Covino, Sandra (2019). Linguistica e nazionalismo tra le due guerre mondiali. Scienza e ideoIogia negli epigoni ascoliani. (271 p.; Percorsi). Bologna: II Mulino. ISBN 978-88-15-28387-0.

\title{
Giorgio Cadorini
}

Il libro presenta il rapporto tra i linguisti italiani attivi tra le due guerre e la politica nazionalista italiana. Mostra come il nazionalismo e il centralismo in Italia, confusi con il patriottismo, non siano iniziati solo dopo la consegna del governo al fascismo, nonché come i nazionalismi abbiano limitato la ricerca linguistica in Europa nella prima metà del XX secolo e nei decenni precedenti.

Sandra Covino, che lungo tutta la sua carriera scientifica ha dedicato molte energie alla ricostruzione della storia della linguistica e della filologia italiane del XIX e XX secolo, presenta le posizioni nella glottologia e nella dialettologia italiane dell'epoca in esame attraverso l'analisi degli scritti pubblicati e la corrispondenza di alcuni luminari, che possiamo suddividere in due generazioni: quella nata intorno alla metà del XIX secolo con Hugo Schuchardt (1842-1927), Francesco D’Ovidio (1849-1925), Carlo Salvioni (1858-1920) e quella nata dopo il 1870, rappresentata da Matteo Bartoli (1873-1946), Giulio Bertoni (1878-1942), Giuseppe Vidossi (1878-1969), Clemente Merlo (1879-1960), Ugo Pellis (1882-1943) e Carlo Battisti (1882-1977). Schuchardt, non essendo italiano, al volume offre piuttosto l'occasione di evidenziare gli aspetti conflittuali nelle relazioni internazionali, così come gli sforzi di riconciliazione dopo la fine della I Guerra Mondiale. Inevitabilmente nel libro ricorre il nome di Graziadio Isaia Ascoli (1829-1907), punto di riferimento metodologico per entrambe le generazioni.

I temi trattati interessano naturalmente un pubblico specialistico, sebbene diversi spunti riguardino la politica dello stato italiano e la collaborazione tra fascismo e ceti colti in generale, cioè argomenti non ristretti alla linguistica. Per- ciò la lettura, soprattutto se integrata con alcuni approfondimenti individuali sugli aspetti della storia europea ed italiana, potrà essere particolarmente arricchente, magari stimolante, per quelli tra i linguisti nati dopo gli anni Settanta che conoscono forse meglio l'assassinio del presidente Kennedy e la Guerra del Vietnam piuttosto che la storia italiana dal 1861 al 1945. Il libro, infatti, cita anche aspetti della storia della linguistica in Italia che finora non sono stati trattati esplicitamente, non necessariamente perché scomodi, ma piuttosto perché già noti a chi era vissuto in quell'epoca o nel periodo immediatamente successivo.

Dal libro i filologi classici e gli storici dell'antichità potranno ricavare informazioni sul pensiero politico e la visione della società attivi in studiosi che hanno prodotto teorie importanti nella ricostruzione del latino parlato, sulla permanenza dei sostrati preromani, sul ruolo dei caratteri etnici, in alcuni casi proiettando inconsapevolmente nel passato i comportamenti loro contemporanei. Tra gli storici è ancora oggi fortemente presente la tendenza a proiettare l'imperialismo ed il colonialismo del XIX secolo sull'interpretazione dell'espansione politica e culturale romana, i quali si riflettono nella linguistica con l'idea di una latinizzazione strettamente dipendente dall'occupazione militare del territorio.

Oggi vediamo altri fenomeni espansivi dell'Unione Europea, della NATO, della lingua inglese, dell'economia cinese (penso in particolare all'Africa), vediamo movimenti migratori, che stimolano a interpretare in altra maniera anche il mondo antico. Perciò sarebbe importante capire meglio cosa delle teorie fondanti delle discipline umane, nate proprio tra Ottocento 
e Novecento, sia attribuibile al contesto culturale di quei secoli e cosa aderisca strettamente ai documenti antichi che abbiamo a disposizione nonché ai dati che possiamo ricavare dalla linguistica comparata.

L'esposizione è suddivisa in quattro sezioni. Comincia descrivendo sinteticamente il contesto italiano ed europeo del dibattito linguistico. Basilare per la generazione nata dopo il 1870 fu la scissione tra i continuatori più fedeli di Ascoli, il fondatore della linguistica scientifica in Italia, ed il gruppo innovatore che elaborò la neolinguistica. I neolinguisti, sostanzialmente, invece che superare il positivismo neogrammatico avanzando sulla strada dello strutturalismo, avevano fatto un passo indietro verso l'idealismo, nella versione aggiornata da Benedetto Croce e Giovanni Gentile, senza trascurare il contributo di Karl Vossler.

Un postulato che, invece, per tutto il periodo in esame prevalse in tutta l'Europa, dunque non solo in Italia, è il principio che la lingua madre determini l'appartenenza nazionale, arrivando fino a stabilire la parentela tra nazioni sulla base della parentela linguistica. Questo postulato dà alla linguistica un peso politico che le impedisce di essere pienamente obbiettiva e neutrale.

Ciò si vide bene quando Ascoli applicò alla classificazione linguistica i concetti elaborati dapprima per la geografia da Carl Ritter, che ha stabilito che si possono individuare dei geotipi in base alla particolare combinazione di un insieme di caratteri. Tradotto in termini linguistici, ciò significa che, per distinguere una varietà linguistica da altre, non è necessario che presenti un singolo tratto suo esclusivo, ma occorre piuttosto che presenti una combinazione esclusiva di tratti. Essi si possono trovare anche in altre varietà, ma combinati con altri tratti che nella prima varietà non ci sono e in mancanza, invece, di altri tratti rilevati nella prima varietà.

Applicando questi concetti, nel biennio 1873-1874 Ascoli definì due nuove varietà lin- guistiche: il francoprovenzale e il retoromanzo (da lui chiamato «ladino»). In Francia, sulla base dell'equazione 1 lingua $=1$ nazione, $\mathrm{ci}$ fu una violenta reazione che respingeva l'evidenza scientifica. In Italia la reazione non fu immediata, in cambio aperse una discussione, chiamata Questione Ladina, che dura ancora oggi (p. 42, n. 20). Nel periodo trattato dal libro la discussione fu particolarmente vivace, proprio perché a parere di molti metteva in dubbio i confini raggiunti dall'Italia con la vittoria della I Guerra Mondiale. Il maggiore oppositore dell'idea di separare gli idiomi retoromanzi dal complesso dei dialetti italiani fu senza dubbio Carlo Battisti, che era nato a Trento ancora nel periodo dell'amministrazione austriaca. Pare che proprio per difendere il carattere linguistico (dunque, per lui, nazionale) prettamente italiano della regione natale sia arrivato ad intervenire pesantemente sul testo di un manoscritto di cui curò l'edizione (p. 55).

Battisti non fu l'unico a provenire da terre che alla sua nascita non erano parte del Regno d'Italia. Anzi, si nota che Sandra Covino dedica intelligentemente una grande attenzione proprio a personaggi che erano nati nell'Impero Austro-Ungarico (oltre a Battisti, gli Istriani Bartoli e Vidossi; tutta l'Istria fu amministrata dal Regno d'Italia dal 1918 fino alla conclusione della II Guerra Mondiale) e allo Svizzero Carlo Salvioni.

Anche Bartoli dedicò molta attenzione alla "difesa" del "suo" confine di stato, quello orientale. Si spinse addirittura fino ad approvarne la collocazione ben oltre il confine linguistico. Come Battisti, ebbe la tendenza ad intervenire sui documenti linguistici, in particolare sui materiali dell'isola di Veglia (di fronte a Fiume). Ebbe anche la tendenza a manipolare le parole di Ascoli, attribuendogli idee sciovinistiche e antidemocratiche (p. 69).

A questi tentativi di Bartoli si oppose recisamente Clemente Merlo, cui è dedicata la III 
sezione del libro. La scelta di approfondire la sua esperienza è decisamente indovinata, perché Merlo rappresenta molto bene diverse fasi che attraversarono più studiosi italiani della sua generazione. L'aspetto che qui mi preme sottolineare è l'illusione di servirsi del fascismo senza esserne coinvolti. Tuttavia Merlo, come altri, rimase fedele alla scienza anche quando contrastava con gli ideali o era scomoda per il potere. Come linguista, questo suo restare se stesso lo distingue dalla maggioranza degli attivi sostenitori del fascismo, ma nel quadro più generale degli studiosi (pensiamo, per esempio, a Enrico Fermi) e non solo degli studiosi, riflette gli atteggiamenti di molti altri, in particolare di coloro che costituirono quella che potremmo definire una "zona grigia" tra il fascismo e l'antifascismo.

Conclude il volume un'appendice dedicata alla corrispondenza tra Schuchardt e D'Ovidio. Anche se questi due linguisti ci riportano indietro nel tempo rispetto al culmine del fascismo (morirono entrambi intorno alla metà degli anni Venti vicini agli ottant'anni di età), l'aver posto l'analisi dell'evoluzione delle loro opinioni alla fine dell'esposizione si mostra una scelta particolarmente efficace. Infatti comporta un ripasso dei temi trattati nelle sezioni precedenti, partendo dall'Ottocento, per seguire poi dettagliatamente le fratture nei rapporti personali provocate dalla "Guerra degli Spiriti" combattuta dagli intellettuali parallelamente alle trincee della I Guerra Mondiale, e quindi osservare il processo di riavvicinamento, che non fu mai totale, al ritorno della pace. In questo modo siamo aiutati a fare una sintesi dopo le analisi molto precise e dettagliate contenute nei capitoli precedenti.

Il libro è importante per i linguisti, perché evidenzia un fatto importante in generale nelle discipline umanistiche: difficilmente possono essere considerate politicamente neutrali. Più facile è per il ricercatore conseguire la neutralità nel descrivere la documentazione a dispo- sizione, più difficile rendersi conto dei condizionamenti sociali cui è soggetto nell'esporli e nell'interpretarli. Essere razzisti nella prima metà del XX secolo in Europa era normale, nazionalisti normalissimo (ma non obbligatorio, molti seppero non essere né l'uno né l'altro). La Questione Ladina e lo studio della Romània adriatica orientale di quell'epoca mostrano come quelle ideologie influissero sull'interpretazione dei dati linguistici.

Un altro stimolo molto positivo è la dimostrazione che è sempre fondamentale riesaminare i documenti originali, che non ci si può accontentare delle sintesi elaborate nei decenni precedenti. La corrispondenza epistolare tra gli studiosi, i discorsi tenuti durante le cerimonie, gli scritti non specialistici sono analizzati e confrontati tra di loro da Sandra Covino con estrema precisione e correttezza. Il libro ciononostante non fa perdere il lettore nella congerie dei materiali, perché i commenti e le sintesi offrono dei chiari fili conduttori che aiutano a riconoscere i punti centrali della problematica e li integrano nel contesto più largo del pensiero e delle opere elaborati in quell'epoca, in modo che il lettore ricollega facilmente le informazioni alle sue conoscenze precedenti e le arricchisce.

Se vogliamo cercare un elemento da proporre per completare il quadro esposto nel volume, un elemento non indispensabile che si sarebbe potuto mettere in luce è il fatto che chi visse in quei decenni aveva visto molti spostamenti di confine, aveva visto scomparire imperi e nascere numerosi nuovi stati. Oggi molti di noi tendono a dimenticarsi che i confini non sono una linea sul terreno, ma una pura astrazione. Per quel che riguarda il dibattito sull'identità nazionale, invito a pensare per quell'epoca alle questioni legate a Cechi, Slovacchi, Ruteni, Cecoslovacchi e Catalani.

A questo proposito non possiamo tralasciare i numerosi israeliti che si trovarono sospinti 
nel ruolo di membri della nazione ebraica. Nel libro il tema è accennato, tra l'altro nel caso di Terracini: volontario decorato nella I Guerra Mondiale, giura fedeltà al regime fascista nel 1931 (si deduce dal fatto che allora non fu espulso dall'università di Milano statale dove insegnava) e in cambio ne viene perseguitato a partire dal 1938 (Merlo gli espresse solidarietà, § 2.3. alle pp. 116-119).

Riguardo al rapporto lingua-nazione, il volume tratta frequentemente il tema riportandone l'attualità nell'epoca in esame. Pare troppo netto il giudizio che esprime presentando come superato il concetto che la lingua sia determinante per l'identità nazionale, in quanto struttura portante della visione del mondo dei singoli parlanti. Gli autori citati dall'autrice (p. 187), però, non parlano della relazione tra lingua e visione del mondo, bensì dello stato-nazione monolingue.

A p. 189 è citata la Dichiarazione universale sui diritti linguistici emanata a Barcellona nel 1996, come sostegno della posizione che vada respinta l'idea della lingua come segno di appartenenza nazionale e come discriminante nazionale. Eppure la dichiarazione di Barcellona proclama la stretta interconnessione tra il gruppo linguistico, il suo territorio storico e la sua cultura; afferma l'obbligo di chi si trasferisce sul territorio dove si usa storicamente una lingua di impararla; quindi, sotto il titolo II, sezione V, mette in stretta relazione lingua e cultura.

La soluzione proposta alla crisi dello stato-nazione monolingue non è, allora, la messa in discussione della nazione, bensì la messa in discussione dello stato monolingue, che deve prendere atto che non è possibile tracciare dei confini in maniera tale da includere una sola nazione (come affermato già dallo storico Eric Hobsbawm, citato in proposito a p. 188) e che perciò i cittadini hanno almeno due livelli di appartenenza: allo stato (plurinazionale) e alla nazione (che può anche essere presente in più stati o solo in una regione dello stato). Aggiungo che va considerato che è un fatto comune che un individuo abbia la cittadinanza di più stati e che partecipi alla vita di più nazioni. Il fatto che la condizione umana più diffusa è il plurilinguismo non significa che la lingua non sia determinante per un individuo nella costituzione della sua visione del mondo, bensì comporta che egli partecipi di più identità e che fruisca di più condizionamenti.

Questo libro parlando del passato tratta temi molto attuali: la crisi dello stato-nazione, il rifugio nel nazionalismo esclusivista, il razzismo come capro espiatorio, il negazionismo. Fondamentale è l'insegnamento che nemmeno la linguistica costituisce un rifugio dalle tensioni politiche, con il correlato che gli accademici non costituiscono una razza eletta.

Il volume è rilegato in brossura, la stampa è molto accurata anche nelle 79 note, rarissimi i refusi. Molte le citazioni in lingua originale con traduzione in nota, inclusi numerosi brani inediti di corrispondenza epistolare. Conclude il libro l'indice dei nomi, molto utile e comodo da consultare.

Dott. Giorgio Cadorini, Ph.D. / giorgio@cadorini.org

Department of Classical Studies

Masaryk University, Faculty of Arts

Arna Nováka 1, 60200 Brno, Czech Republic

This work can be used in accordance with the Creative Commons BY-SA 4.0 International license terms and conditions (https://creativecommons.org/licenses/by-sa/4.0/legalcode). This does not apply to works or elements (such as image or photographs) that are used in the work under a contractual license or exception or limitation to relevant rights. 
